\title{
Diabetic Embryopathy: A Developmental Perspective from Fertilization to Adulthood
}

\section{Castori}

Division of Medical Genetics, Department of Molecular Medicine, Sapienza University, San Camillo-Forlanini Hospital, Rome, Italy

\section{Key Words}

Blastogenesis · Diabetes mellitus · DOHeD • Epigenetics • Spontaneous abortion - VACTERL

\begin{abstract}
Maternal diabetes mellitus is one of the strongest human teratogens. Despite recent advances in the fields of clinical embryology, experimental teratology and preventive medicine, diabetes-related perturbations of the maternofetal unit maintain a considerable impact on the Healthcare System. Classic consequences of prenatal exposure to hyperglycemia encompass (early) spontaneous abortions, perinatal death and malformations. The spectrum of related malformations comprises some recurrent blastogenic monotopic patterns, i.e. holoprosencephaly, caudal dysgenesis and oculoauriculovertebral spectrum, as well as pleiotropic syndromes, i.e. femoral hypoplasia-unusual face syndrome. Despite this, most malformed fetuses display multiple blastogenic defects of the VACTERL type, whose (apparently) casual combination preclude recognizing recurrent patterns, but accurately testifies to their developmental stage at onset. With the application of developmental biology in modern medicine, the effects of diabetes on the unborn patient are expanded to include the predisposition to develop insulin resistance in adulthood. The mechanisms underlying the
\end{abstract}

transgenerational correlation between maternal diabetes and proneness to adult disorders in the offspring remain unclear, and the epigenetic plasticity may represent the missing link. In this scenario, a development-driven summary of the multifaced consequences of maternal diabetes on fertility and child health may add a practical resource to the repertoire of available information on early stages of embryogenesis.

Copyright $\odot 2012$ S. Karger AG, Basel

Since the early 1900s, maternal diabetes mellitus (DM) has been known as a potent human teratogen [White, 1937]. After the therapeutic availability of insulin in the 1920 s, consequences of DM on the development of the fetus progressively overcame those to maternal well-being in the perspective of practitioners managing pregnant women with DM [Zabihi and Loeken, 2010]. The 'epidemic' diffusion of DM in both developed and developing countries [Yajnik, 2009] and the increasing maternal age at conception occurring in the industrialized world are expanding the impact of diabetic teratogenicity in the field of maternofetal medicine. In addition, despite available preventive strategies to reduce the incidence of DM-induced malformations, nearly two-thirds of pregnancies in diabetic mothers are unplanned; a fact that

\section{KARGER}

Fax +4161306 1234

E-Mail karger@karger.ch

www.karger.com (c) 2012 S. Karger AG, Basel

$1661-8769 / 13 / 0042-0074 \$ 38.00 / 0$

Accessible online at:

www.karger.com/msy
Marco Castori, MD

Division of Medical Genetics, Department of Molecular Medicine

Sapienza University, San Camillo-Forlanini Hospital

Circonvallazione Gianicolense 87, IT-00152 Rome (Italy)

E-Mail mcastori@scamilloforlanini.rm.it 
challenges any evidence-based approach. Recently, the evolving scientific thinking envisages the fact that the echoes of a prenatal exposure to maternal DM may move far beyond birth. In fact, maternal hyperglycemia can permanently affect the offspring's metabolism by increasing the risk for some noncommunicable adult disorders, which develop as late consequences of an adverse intrauterine environment [Yajnik, 2009]. In line with this new scenario, it is expected that medical/clinical genetics consultation will be increasingly requested by diabetic women after ultrasound and/or postnatal detection of (potentially) teratogen-induced malformations. Such specialized support may help in etiologically interpreting the findings and/or receiving up-to-date education for preventing both fetal and later life reverberations in future pregnancies.

The aims of this review are (i) to illustrate the spectrum of malformations related to maternal DM and possible associative presentations, (ii) to offer a comprehensive overview of the effects of DM on the maternofetal unit, and (iii) to give to the practicing clinical geneticist rapidly consultable information to proficiently take part in the multidisciplinary team managing the preconception and prenatal risks of DM.

\section{Types of Diabetes and Types of Risks}

Historically, the teratogenic risk related to maternal diabetes has been investigated in DM type 1 (DM1), and the focus of interventions for reducing the rate of birth defects has been on this condition [Kitzmiller et al., 1996]. In the past, the rationale was that, as the teratogenic potential of DM is directly related to glucose and glycohemoglobin serum levels and inversely related to the gestational age at first exposure, the maximal risk occurs in women who develop diabetes years before conception. At that time, this was most commonly the case of patients with DM1. In the succeeding decades, maternal age at conception has progressively increased, and the number of women with DM type 2 (DM2) at the time of conception increased exponentially. Therefore, in the last years, much attention has been placed on women with DM2, and recent evidence demonstrates overlapping rates of major congenital malformations, stillbirth and neonatal mortality in pregestational DM1 and DM2 [Balsells et al., 2009]. In addition, DM2 in pregnancy demonstrates unique implications, such as responsiveness to drugs (e.g. statins) with (unclear) teratogenic potential, the danger of being unrecognized before pregnancy, poor nutrition, and the association with comorbidities, including advanced maternal age, obesity, lipid peroxidation, and lowgrade inflammation [Kitzmiller et al., 2010].

At variance with pregestational DM, the rate of major malformations is only slightly increased in the offspring of mothers with gestational DM (GDM; i.e. hyperglycemia firstly diagnosed during pregnancy). In GDM, the relative risk of birth defects is only slightly increased, ranging from 1.16-1.40, depending on the study type [Balsells et al., 2012]. However, the population of women with GDM is more heterogeneous than with DM1 or DM2. In fact, there are reasonable concerns regarding the accuracy of diagnosis in at least a proportion of GDM women, who may be affected by a neglected prediabetic status prior to pregnancy and who are first diagnosed only after conception. This GDM subgroup is likely at higher risk for perinatal complications. Accordingly, among pregnancies complicated with GDM, the association with birth defects is generally limited to infants from women with pre-pregnancy overweight status (i.e. body mass index $\geq 25$ ), which is considered a marker of insulin resistance [Correa et al., 2008].

In conclusion, the highest risk for malformations is reported for women with pregestational DM, irrespective to the type. An intermediate risk is reported for overweight women developing GDM, while pregnant women with GDM, but lacking prediabetic markers, experience perinatal outcomes similar to the general population. However, GDM is not free from other fetal complications, such as macrosomia and glucose metabolic derangement, related to late in utero exposure to maternal hyperglycemia.

\section{Maternal Diabetes and Malformations}

It is now clear that the effects on the embryofetal integrity are overlapping between DM1 and DM2. The spectrum of malformations related to maternal DM is wide, and can be associated with the involvement of most body parts, including the cardiac and outflow tract, craniofacial structures, as well as the central nervous, gastrointestinal, musculoskeletal, and urogenital systems (fig. 1). Although the teratogenic mechanisms leading to perturbed organogenesis are still incompletely understood in DM (see 'Mechanisms of Teratogenesis in Maternal Diabetes'), the types and severity of observed phenotypes, as well as the timeframe correlations between prenatal exposure to maternal hyperglycemia and embryological staging, suggest that DM acts early in gesta- 
Fig. 1. Some examples of malformations related to maternal diabetes. A Anal atresia in a 22-week-old fetus aborted for multiple malformations at ultrasound. B Radiograph of the same fetus showing sacral hypoplasia and narrowing of the ischial bones (i.e. caudal disgenesis). C X-ray examination of a 23-week-old fetus (for complete clinical details, see Castori et al. [2010]) with multiple vertebral segmentation defects; this fetus had a clinical diagnosis of VACTERL association. D A 21-week-old fetus with sirenomelia. E Newborn with apparently isolated right microtia, born to a diabetic mother with early-onset diabetes type 2. F Left thumb hypoplasia in a VACTERL boy (for complete clinical details, see Castori et al. [2008]). G Typical facial features in a girl with femoral hypoplasia-unusual face syndrome born to a mother with diabetes type 1. $\mathbf{H}$ Pelvic anomalies in the same patient.
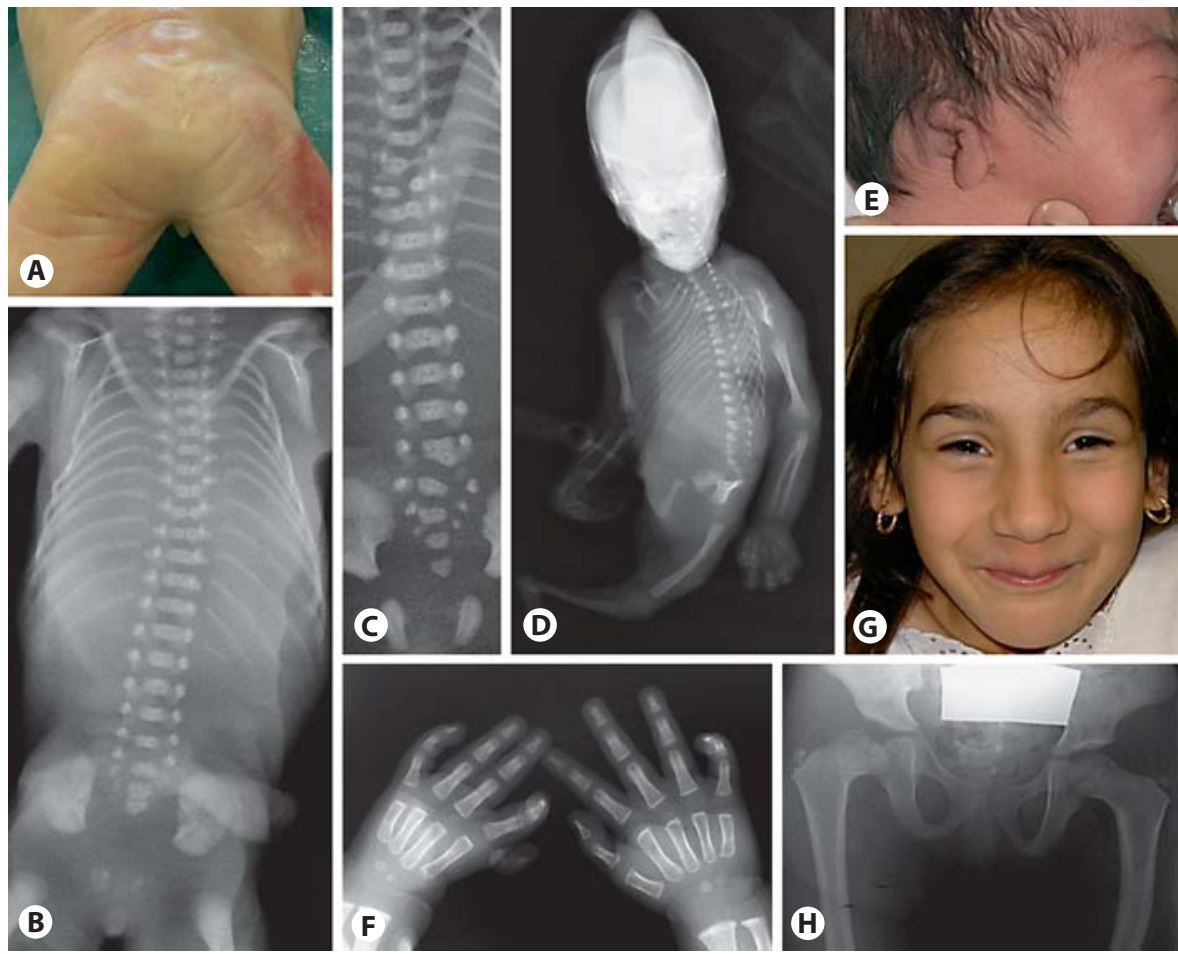

tion. Accordingly, a great proportion of malformations occurring in infants from diabetic mothers are blastogenic in origin. In other words, they originate from an environmental 'hit' acting during the first 4 weeks of gestation (i.e. blastogenesis) according to the developmental field defect theory [Opitz et al., 2002]. Table 1 summarizes the various types of birth defects associated with DM. The extreme variability of the relative risk for each tabulated malformation indicates that the various organs have different thresholds of susceptibility to the teratogenic potential of DM. Notably, the relative risk for most malformations is higher in infants with multiple birth defects [Correa et al., 2008]. This implies that, for congenital anomalies reported in infants born to mothers with pregestational DM, correlation with maternal hyperglycemia is higher in individuals with multiple malformations ( $\sim 90 \%)$ than in isolated defects $(\sim 70 \%)$ [Correa et al., 2008]. Therefore, recently recorded epidemiological data confirm the concept that DM affects early embryogenesis. In fact, in this stage, a single perturbation may influence the formation of developmental fields destined to form different structures simultaneously.

Such devastating consequences of DM on embryogenesis moved many research groups to investigate the existence of a recognizable malformation pattern among in- fants from diabetic mothers. Since the 1970s, caudal dysgenesis (alternatively and improperly termed 'caudal regression syndrome') has shown the most striking association with DM [Kucera, 1971; Pedersen and MølstedPedersen, 1978]. Although the strength of this association has been subsequently downsized [Martínez-Frías, 1994], caudal dysgenesis remains the single malformation with the highest relative risk in diabetic embryopathy (table 1). However, given the rarity of caudal dysgenesis in the general population, the occurrence of this malformation in infants from a diabetic mother is very low $(\sim 1 \%)$ and does not differ significantly from that reported for holoprosencephaly [Barr et al., 1983]. Among the regional or nonblastogenic recognizable patterns associated with pregestational DM, there are holoprosencephaly [Barr et al., 1983], femoral hypoplasia-unusual face syndrome [Johnson et al., 1983] and oculoauriculovertebral spectrum [Ewart-Toland et al., 2000; Wang et al., 2002]. All these presentations show strong correlation with maternal hyperglycemia, and this feature should be always investigated in regards to adequate prevention strategies in future pregnancies. Nevertheless, excluding the cases showing such recurrent phenotypes, $>90 \%$ of infants from diabetic mothers display apparently nonspecific associations. The lack of a prevailing recognizable pattern cor- 
Table 1. Spectrum of malformations observed in infants/fetuses born to mothers with pregestational diabetes

\begin{tabular}{|c|c|c|}
\hline Birth defect & Relative frequency (RF) & Reference(s) \\
\hline \multicolumn{3}{|l|}{ Nonblastogenic malformations } \\
\hline Bifid tongue & undetermined $^{\mathrm{a}}$ & Comess et al., 1969; James et al., 2007 \\
\hline Cleft lip \pm cleft palate & $2.92^{\mathrm{b}}$ & Correa et al., 2008 \\
\hline Facial dysmorphism & undetermined $^{\mathrm{c}}$ & Johnson et al., 1983 \\
\hline Hydrocephaly & $8.80^{\mathrm{b}}$ & Correa et al., 2008 \\
\hline \multicolumn{3}{|l|}{ Blastogenic malformations } \\
\hline Anorectal atresia/stenosis & $2.81-4.70^{\mathrm{b}}$ & Frías et al., 2007; Correa et al., 2008 \\
\hline Caudal dysgenesis & $53.00-200.00$ & Kucera, 1971; Martínez-Frías, 1994 \\
\hline Congenital heart defects & up to $18.24^{\mathrm{d}}$ & Correa et al., 2008; Lisowski et al., 2010 \\
\hline Costovertebral segmentation defects & $26.30-39.30^{\mathrm{e}}$ & Martínez-Frías, 1994 \\
\hline Neural tube defects & $2.90^{\mathrm{b}, \mathrm{g}}$ & Martínez-Frías, 1994 \\
\hline Renal adysplasia & $3.10-10.43^{\mathrm{h}}$ & Bánhidy et al., 2010; Davis et al., 2010 \\
\hline Sirenomelia & undetermined $^{\mathrm{b}, \mathrm{i}}$ & Valenzano et al., 1999; Castori et al., 2010 \\
\hline Thymus aplasia & 29.62 & Frías et al., 2007 \\
\hline Urorectal septum malformation & undetermined $^{\mathrm{a}}$ & Gripp et al., 1999 \\
\hline
\end{tabular}

\footnotetext{
a Single reports only.

${ }^{\mathrm{b}}$ Not all studies replicate similar results [Bánhidy et al., 2010].

${ }^{c}$ Including the facial phenotype typical of 'femoral hypoplasia-unusual face syndrome'.

d Conotruncal malformations, heterotaxia, univentricular heart and atrial ventricular septal defects being the most com-

e The lower end of the range relates to upper spine malformations, while the upper end refers to the lower spine.

${ }^{\mathrm{f}}$ Mainly comprising focal proximal femoral hypoplasia and hallucal polydactyly. The RF for hallucal polydactyly is fixed to 3.62 [Frías et al., 2007].

g A more recent study [Correa et al., 2008] confirms an increased risk for anencephaly and craniorachischisis $(\mathrm{RF}=3.39)$,
} mon.

and encephalocele $(\mathrm{RF}=2.09)$, while spina bifida $(\mathrm{RF}=0.75)$ does not seem more common among children from diabetic mothers.

${ }^{\mathrm{h}}$ Renal adysplasia includes a wide spectrum of different kidney malformations, including unilateral and bilateral aplasia, hypoplasia and multicystic disease. Various studies have investigated the RF for specific phenotypic subtypes, comprising bilateral renal agenesis/hypogenesis $(\mathrm{RF}=11.91)$ [Correa et al., 2008] and multicystic dysplastic kidneys $(\mathrm{RF}=6.15)$ [Frías et al., 2007].

${ }^{i}$ The association between sirenomelia and maternal diabetes is still debated [Kalter, 1993]; no systematic study testing this hypothesis has been performed to date; however, the strength of the presumed association is likely weaker than with caudal dysgenesis, as maternal diabetes is registered in only $1.7 \%$ children with sirenomelia [Duncan et al., 1991].

relating with maternal DM has been explained by a multifactorial basis for DM-induced embryopathy [Reece et al., 1996]. This hypothesis implies that complex underlying metabolic disorders that are associated with DM increase the chance that different signal transduction pathways and morphogenetic processes might be disturbed.

Accumulated data indicate that malformed infants from diabetic mothers often present with multiple blastogenic malformations. The variability in the combination of blastogenic defects is theoretically unlimited in humans [Opitz, 1993]. Previous reports of complex malformation patterns in the offspring of women with pre- gestational DM demonstrate that this rule of thumb is also valid in diabetic embryopathy (table 2). Therefore, the diabetic embryopathy may be more correctly interpreted as an etiological subgroup of defects of blastogenesis presenting with a wide variety of monotopic and polytopic developmental field defects. In contrast with the overall rarity (as opposed to their relative specificity) of some blastogenic malformations, such as caudal dysgenesis, holoprosencephaly and oculoauriculovertebral spectrum, it may be presumed that most infants of diabetic mothers present the variable association of costovertebral segmentation defects (V), ano-rectal stenosis/ 
Table 2. Published examples of (possibly recurrent) polytopic developmental field defects in infants/fetuses of mothers with pregestational diabetes

\begin{tabular}{ll}
\hline Phenotype & Reference(s) \\
\hline Cardiac and visceral heterotaxia & Frías et al., 2007; Lisowski et al., 2010 \\
DiGeorge sequence and caudal dysgenesis & Dentici et al., 2012 \\
$\begin{array}{l}\text { DiGeorge sequence and kidney agenesis } \\
\text { 'Expanded' oculoauriculovertebral spectrum }\end{array}$ & Gosseye et al., 1982; Wilson et al., 1993; Novak and Robinson, 1994; Digilio et al., 1995 \\
Holoprosencephaly and caudal dysgenesis & Chen et al., 2005 \\
VACTERL association & Boutte et al., 1985; Loffredo et al., 2001; Castori et al., 2008
\end{tabular}

a In addition to the typical oculoauriculovertebral spectrum, among the reported patients, there are subjects showing extra-regional features, such as central nervous system malformations, micro-/anophthalmia, radial ray defects, congenital heart defects, and anal stenosis/atresia.

atresia (A), complex congenital heart defects (C), renal adysplasia (R), and longitudinal limb defects (L), along with various neural tube defects (table 1 ). An early study by Driscoll [1965] also showed a slight increase in the frequency of single umbilical artery in the placentae of pregnancies from diabetic mothers. In line with this, the "nonspecific' combination of VACTERL features seems to emerge as a common presentation of diabetic embryopathy. At the moment, the lack of universally accepted diagnostic criteria for VACTERL association [Hall, 2010] hampers the possibility to test the existence of a phenotypic subtype, within the clinical spectrum of VACTERL association, which may be more specific for diabetic embryopathy. However, indirect data from table 1 envisage that the combination of 2 or more VACTERL features combined with an open neural tube defect and lacking tracheal-esophageal malformation (which, indeed, is not found to be associated with maternal diabetes; see, for example, Correa et al. [2008]) could be the most typical presentation of diabetic embryopathy. If confirmed by future studies, a more consistent correlation between maternal DM and VACTERL association may shed further light on the mechanisms regulating early embryogenesis and stimulate more evidence-based strategies for investigating the theory of developmental field defects in humans.

\section{Maternal Diabetes, Abortion and Perinatal Deaths}

Gestational consequences of DM are not limited to birth defects. An increased rate of spontaneous abortions in prospective mothers with pregestational DM was known since the 1950s [Kalter, 1987]. Various studies were performed in the following 5 decades, and the mean additional risk was fixed to $\sim 15 \%$ [Kitzmiller et al., 1996]. Such percentage roughly doubles the rate of spontaneous abortion in the general population and should be added to the $\sim 25 \%$ chance of 'chemical pregnancies' (i.e. early miscarriages diagnosed by positive pregnancy test before the woman's period) likely shared by both populations [Miller et al., 1980]. Consequently, more than half of conceptions in women with pregestational DM interrupt spontaneously. Similarly to the risk of birth defects, also the rate of spontaneous abortions is influenced by the pre-pregnancy state, comprising glucose and glycohemoglobin serum levels and maternal weight [Kitzmiller et al., 1996; Yogev et al., 2010]. These notions are crucial in term of preventive strategies (see 'Principles of Prevention').

Restricted embryofetal vitality in maternal DM may have different clinical presentations, including early spontaneous abortions, late (i.e. second-trimester) spontaneous abortions and stillbirths/neonatal deaths. Such a heterogeneity reflects the protean effects of DM on the maternofetal unit. In an early study, Mills and coworkers [1988] demonstrated that in diabetic mothers 30\% of spontaneous abortions happen before the 8th gestation week, $58 \%$ at $8-11$ weeks and only $12 \%$ at $12-19$ weeks. In other words, in maternal DM, pregnancies losses most commonly ( $\sim 90 \%)$ occur in the first trimester. Furthermore, advanced maternal and non-Caucasian ethnicity are associated with the risk of spontaneous abortions in diabetic mothers. The mechanisms leading to early pregnancy loss in maternal DM have not been studied in depth. Greene et al. [1989] showed that chromosome abnormalities, 'blighted ova' (i.e. development of the chorioamnion without the embryo) and absent fetal cardiac 
activity are common presentations of first-trimester pregnancy losses in DM. However, such excess of early abortions may be also determined by metabolic embryopathy [Kitzmiller et al., 1996], which, more recently, seems to be partly related to an excess of oxidative stress in diabetic pregnancies [Damasceno et al., 2002].

Second-trimester abortions are likely underlined by different pathogenic processes compared to early losses. This happens in the general population as well as in diabetic pregnancies. In this stage, the role of abnormal organogenesis and placentation is presumably more relevant. Therefore, it is expected that severe birth defects (such as complex congenital heart defects), placental insufficiency, maternal metabolic derangement (e.g. maternal ketoacidosis in DM1), and fetal hypoxia due to premature rupture of the membranes are leading causes of late pregnancy losses in DM. However, literature data are scanty on this point.

More studies are available investigating the rate and spectrum of causes of stillbirths and neonatal deaths in maternal DM [Melamed and Hod, 2009]. Perinatal deaths are still a challenge in the management of diabetic pregnancies, as they have a frequency as high as $6.6 \%$, with a relative risk of 9.0 compared to the general population [Clausen et al., 2005]. Among the possible factors causing stillbirths (i.e. in utero death), in maternal DM, most of them, including malformations, placental insufficiency and maternal ketoacidosis, are shared with those related to second-trimester abortions. However, while no study to date has been published investigating the exact role of placentation and maternal metabolic derangement on in utero vitality, lethal malformations account for only $16-$ $28 \%$ of stillbirths [Platt et al., 2002; Jensen et al., 2004; Macintosh et al., 2006]. Conversely, fetal consequences of maternal hyperglycemia (i.e. poor glycemic control during the second and third trimesters) are probably the leading factor of stillbirths in diabetic pregnancies, as 50$75 \%$ of the in utero deaths may be attributed to this feature [Lauenborg et al., 2003; Dudley, 2007].

Neonatal deaths are most commonly related to macrosomia (41-62\%) [Jensen et al., 2004; de Valk et al., 2006], prematurity (21-41\%) [Jensen et al., 2004; de Valk et al., 2006], neonatal asphyxia (26.7\%) [Mimouni et al., 1988], pre-eclampsia (12.7-20.6\%) [Hanson and Persson, 1993; Evers et al., 2004], and respiratory distress syndrome (10.6-17\%) [Boulot et al., 2003; Jensen et al., 2004]. On a pathogenic perspective, placental pathology and fetal/neonatal metabolic derangement due to poor metabolic control in the mothers are the leading causes of neonatal death in diabetic pregnancies. Therefore, the influ-

Diabetic Embryopathy: A Developmental Perspective ence of congenital malformations on perinatal survival rate seems small, and this may be also related to the improvement of therapeutic strategies for congenital anomalies. Nevertheless, the increased rate of birth defects in diabetic pregnancies remains a major issue in terms of long-term prognosis and follow-up.

\section{Mechanisms of Teratogenesis in Maternal Diabetes}

As illustrated in previous sections, accumulated data indicate that blastogenic malformations (usually, with multiorgan involvement) and spontaneous abortions of the first trimester are the most common adverse effects of maternal DM on the maternofetal unit. In humans, these observations stand for indirect evidence for identifying [Opitz, 1993; Opitz et al., 2002] the window of the highest susceptibility of the conceptus to maternal hyperglycemia and its teratogenic mediators in blastogenesis (i.e. the first 4 weeks after conception - from fertilization to the end of gastrulation).

Due to ethical limitations to investigations of embryogenesis in humans, animal studies have been performed in order to extrapolate interspecies generalizations. An early confirmation of the above-mentioned clinical feelings comes from Eriksson et al. [1989], who demonstrate a narrow window of susceptibility to maternal DM during gastrulation and neurulation (corresponding to days 18-35 postfertilization in humans) in rats. However, exposure to hyperglycemia may have significant effects also in earlier stages of development. In fact, Pampfer et al. [1990] show that maternal diabetes causes a reduced inner cell mass in rats. On one side, this phenomenon may amplify subsequent teratogenic insults hampering self-recovery of the developing embryo and, thus, contributing in rising the chance of spontaneous abortion and (polytopic) malformation patterns. On the other side, reduction of the inner cell mass could contribute to intrauterine growth restriction (IUGR), a common feature in pregnancies of women with poorly controlled DM and who likely have the highest glucose plasma levels around fertilization. Consequently, the DM teratogenic potential affects the entire pre-embryonic (i.e. blastogenesis) stage. Slight variations in timing of maximal exposure/susceptibility at this stage may have extremely variable consequences in the succeeding developmental phases. This rationale is further confirmed by the evidence that increased maternal glucose levels around early organogenesis (i.e. 5-6 weeks after fertilization in humans) does not correlate with an increased 
rate of major (i.e. blastogenic) malformations, but, instead, causes cleft palate and some nonaxial skeletal malformations in mice. Later exposure to DM is not associated with birth defects at all [Watanabe and Ingalls, 1963; Horii et al., 1966; Deuchar, 1977].

A further level of investigation is the dissection of biochemical mechanisms bridging exposure to an abnormal maternal metabolism to perturbed organogenesis. Many studies have been published investigating the 'fuel-mediated teratogenesis' of DM. Accordingly, the rate of malformations directly correlates with maternal serum levels of glucose, $\beta$-hydroxybutyrate and acetoacetate and their embryotoxic effects are amplified when added in combination [De Hertogh et al., 1991; Ornoy and Zusman, 1991]. Decreased myo-inositol may be a further mediator of teratogenesis in diabetic embryopathy [Hod et al., 1986; Sussman and Matschinsky, 1988]. Deficiency of specific nutrients related to maternal diabetes may contribute to teratogenesis (i.e. 'nutrient-mediated teratogenesis'). Accordingly, insulin-induced hypoglycemia [Hunter and Sadler, 1989; Smoak and Sadler, 1990] and dietary restriction of zinc [Uriu-Hare et al., 1989] correlate with increased rate of malformations in mice and rats, respectively.

The direct correlation between production of reactive oxygen species and glucose levels is a well-consolidated concept [Jain et al., 1989; Brownlee, 2001]. The embryo, in turn, is highly vulnerable to reactive oxygen species because free radical enzymes are not sufficiently expressed until the end of pregnancy [el-Hage and Singh, 1990; Ishibashi et al., 1997]. Consequently, it is reasonable that oxidative stress may mediate hyperglycemia in determining embryonic malformations. Some evidence emerges from the study by Eriksson [1991], who identifies 3 genetically homogenous strands of rats which differ in susceptibility to skeletal malformations in presence of diabetes. This discrepancy is associated with variations at the erythrocyte catalase locus, Cs-1, encoding an enzyme involved in the free radical metabolism. In addition, various studies demonstrate that administration of supplemental antioxidants in rats reduces the incidence of birth defects in the offspring of diabetic mothers [Zabihi and Loeken, 2010]. Therefore, it is likely that increased oxidative stress has a prominent role in mediating maternal hyperglycemia and perturbed embryogenesis.

Increasing evidence indicates that diabetic embryopathy is related to altered expression of developmental control genes [Fine et al., 1999; Zabihi and Loeken, 2010]. Accurate details on altered gene expression are now available for neural tube defects associated with maternal dia- betes [Salbaum and Kappen, 2010]. Inhibition of Pax3 expression is the best known transcriptional effect of maternal diabetes [Fine et al., 1999]. Pax3 significantly affects the development of the neural tube and its pathogenic potential is demonstrated by the existence of the Splotch genotype in mice. In fact, Splotch embryos carry a loss-of-function mutation in Pax3 and the homozygous Splotch genotype causes neural tube defects with $100 \%$ penetrance [Auerbach, 1954]. The link between Pax3 insufficiency and neural tube defects may be represented by an increased apoptotic rate in critical developmental stages [Phelan et al., 1997]. This phenomenon may be mediated by an increase in production of $\mathrm{p} 53$ protein in cells expressing inappropriately low levels of Pax3 [Pani et al., $2002 \mathrm{a}, \mathrm{b}$ ]. The work by Li et al. [2005] elegantly demonstrates that hypoxic stress can reduce Pax 3 expression and, then, is associated with an increased rate of neural tube defects in embryos from diabetic mothers. This evidence adds invaluable data in the attempt to close the 'circle', which traces the various morphological, biochemical and molecular steps bridging maternal hyperglycemia to malformations.

The extreme phenotypic variability and unpredictability of diabetic embryopathy indicates that the etiology of birth defects related to maternal hyperglycemia is multifactorial. The experimental proof that resistance to DM-induced malformations is genetic in origin corroborates this hypothesis [Pani et al., 2002a] as well as the fact that specific dietary habits influence the rate of malformations in the offspring of diabetic mothers. Recently, it has been hypothesized that epigenetic plasticity and its relationships with specific environmental factors, such as diet and metabolic changes, play a prominent role in the development of birth defects in various maternal conditions, including DM [Salbaum and Kappen, 2011]. Preliminary experimental findings are in line with this hypothesis [Salbaum and Kappen, 2012]. Accordingly, most variations in gene expression, which are considered the biological feature most closely related to the developmental perturbations in DM, likely reflect consequences that hyperglycemia and its teratogenic mediators have on the epigenetic regulation mechanisms, comprising DNA methylation, noncoding RNA expression, transcription factor activities, and histone modifications. Figure 2 exemplifies the complex pathogenesis of diabetic embryopathy, which could be considered the result of the interactions among at least 4 major players, including environment as well as maternal, paternal and offspring genomes. The clinical outcome also depends on various intermediate factors, including timing of exposure, se- 


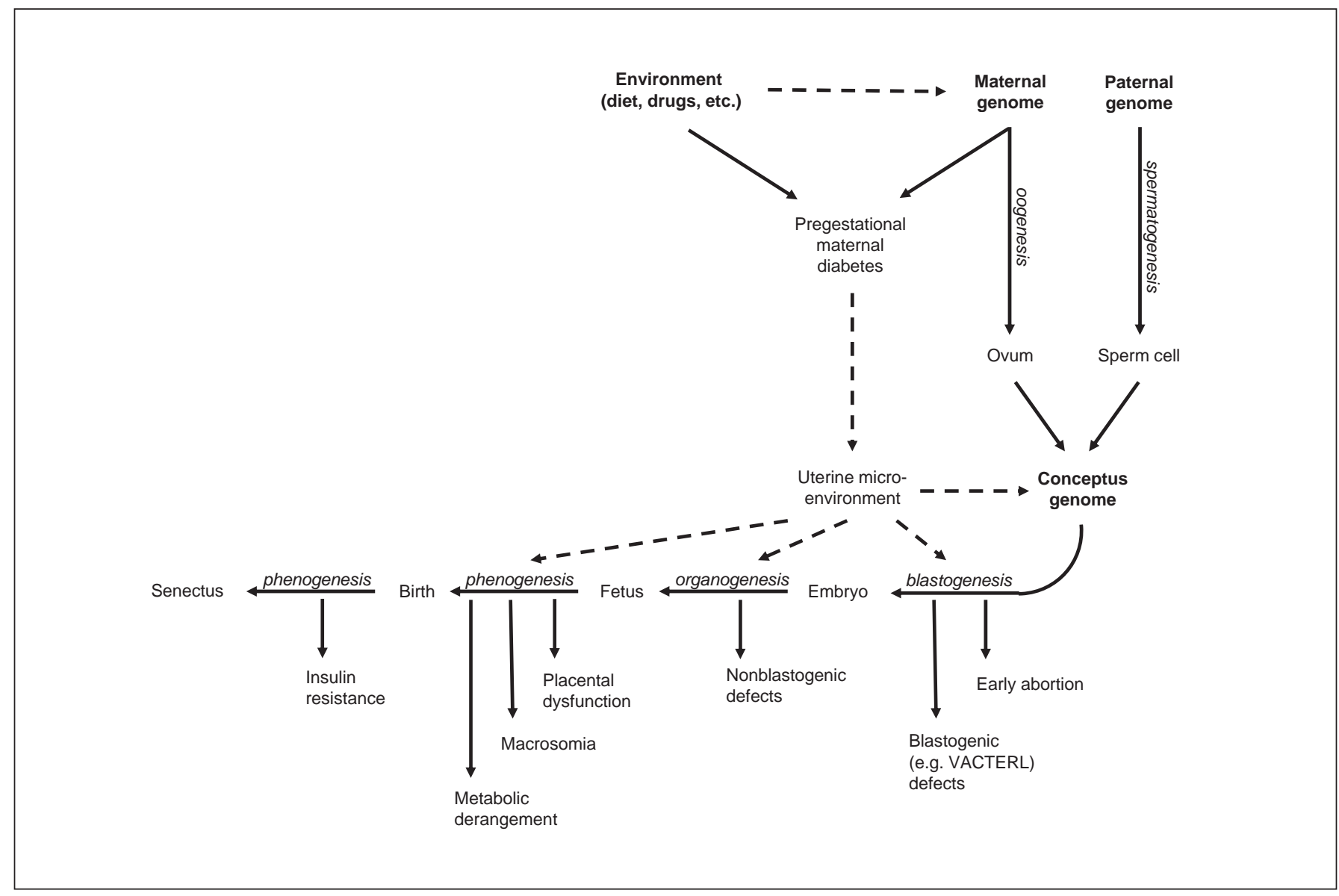

Fig. 2. Interactions among cofactors determining abnormalities in the offspring of mothers with pregestational diabetes mellitus. Dotted lines indicate 'influences'; solid lines indicate progression or consequences.

verity of consequences of maternal diabetes and dietary habits on the intrauterine micro-environment and epigenetic regulation, among others.

\section{Maternal Diabetes and the Future Risk of Adult Diseases in the Offspring}

In the 21 st century, increasing attention is being placed on the long-term influences of an adverse intrauterine environment on the future health of the conceptus once the stage of adulthood has been reached. This field of interest is named 'developmental origins of health and disease' and is based on the assumption that many adult, noncommunicable diseases, such as hypertension, obesity and DM2 (i.e. insulin resistance), could originate from the fetal developmental plasticity to respond to poor in utero conditions [Barker, 2004]. Correlations between poor intrauterine wellness and adulthood diseases are difficult to investigate. IUGR has been considered a clearcut indicator of in utero undernutrition for the conceptus. Accordingly, many studies have investigated the relationships between IUGR and adult-onset metabolic disorders and demonstrated that the former is a predictor for hypertension, diabetes mellitus and obesity in adulthood [Joss-Moore and Lane, 2009]. A simplification of the mechanisms explaining this apparently paradoxical association is that an adverse intrauterine environment causes a premature shifting of the baseline metabolism of the embryo from catabolic to anabolic. Deficient in utero nutrition acts as a selector for those fetuses that carry some acquired and/or inherited adaptation resources. In line with the actual theory of 'adaptation' in evolutional biology, both genetic adaptation and plasticity (i.e. ir- 
Table 3. General risks related to pregestational diabetes

\begin{tabular}{|c|c|c|c|c|}
\hline Feature & Rate in the GP, \% & Relative risk & Absolute risk, \% & Reference \\
\hline Spontaneous abortion & $\sim 15(\text { plus } \sim 25 \%)^{\mathrm{a}}$ & $\sim 2$ & $\sim 30^{\mathrm{b}}$ & Kitzmiller et al., 1996 \\
\hline Malformations at birth & $2-3$ & $2-6$ & $5-15$ & Becerra et al., 1990 \\
\hline Perinatal death ${ }^{c}$ & $0.5-1.8$ & $1.5-9$ & $1.7-10.3$ & Melamed and Hod, 2009 \\
\hline Glucose intolerance $^{\mathrm{d}}$ in the offspring & $\sim 4$ & 2.7 & $\sim 11$ & Clausen et al., 2008 \\
\hline \multicolumn{5}{|c|}{$\begin{array}{l}\text { GP = General population. } \\
\text { a In the general population, } \sim 15 \% \text { conceptions end as sponta- } \\
\text { neous clinical abortion manifesting with cramps, hemorrhages } \\
\text { and/or passage of tissue, while an additional } \sim 25 \% \text { conceptions } \\
\text { are 'chemical pregnancies'. } \\
\text { b The risk of spontaneous abortion is higher only in women } \\
\text { who start pregnancy with elevated glycohemoglobin levels. }\end{array}$} \\
\hline
\end{tabular}

reversible nonheritable changes - epigenetics) may act in the selection of surviving fetuses [Solomons, 2009].

Maternal diabetes (both pregestational and gestational) may be complicated by IUGR as the result of placental insufficiency. Reduced inner cell mass and some major malformations (e.g. congenital heart defects) are further minor contributors to IUGR in maternal DM. In maternal DM, in utero undernutrition could predispose the surviving fetuses to some adult noncommunicable disorders. Accordingly, in recent years, a growing number of studies have been published demonstrating an increased rate of glucose intolerance (eventually, developing into DM type 2) and obesity among adults born from diabetic pregnancies complicated with IUGR, especially if followed by postnatal overnutrition in the very first years of life [Fernández-Morera et al., 2010]. This phenomenon likely displays a strong epigenetic contribution [Fernández-Morera et al., 2010] and rises as the result of acquired but stable modifications of gene expression. In line with this, the long-term consequences (either acquired, or genetic or both) of IUGR in DM may represent the in utero precursor of the 'thrifty' genotype(s) predisposing to DM2. Therefore, in terms of developmental medicine [Crespi, 2011], the long-lasting (i.e. epigenetic) effects of maternal diabetes on gene regulation of the developing embryo, which prospectively become diabetic in (early) adulthood, could act as a selective advantage facilitating diffusion of DM in the past.

Intergenerational connections between glucose intolerance in the pregnant mother and her offspring, prospectively becoming 'diabetic' in adulthood, are strongly based on the plasticity of the epigenome. Reiteration of this process among generations may result in an appar- ently vertical transmission of the disease, presumably, with variable expression and lack of penetrance. This emerging hypothesis, if confirmed by more consistent data, could modify the future approach to the study of 'genetic' contribution to many adult diseases and open new perspectives of prevention and treatment of DM embryotoxicity.

\section{Principles of Prevention}

Despite modern knowledge and the increasing number of resources for reducing the occurrence and early diagnosis of diabetes-related complications, influences of $\mathrm{DM}$ on fertilization and in utero development still represent a considerable clinical problem with a spectrum of risks for the pregnant woman and her offspring (table 3). Nowadays, an affected woman has a wide available range of preventive strategies and diagnostic options to follow for significantly reducing the rate of adverse perinatal outcomes. Table 4 summarizes what is actually considered useful and evidence-based for this purpose.

Direct correlation between maternal glucose levels and, more specifically, with glycohemoglobin allows the refinement of the estimated risk of malformations on the basis of prepregnancy maternal serum markers. In particular, a review of studies published between 1981 and 1994 indicates that the overall chance of major birth defects in the offspring of diabetic mothers is lowest (range: $0.7-4.4 \%$; mean $2.2 \%$ ) for glycohemoglobin levels $<7 \%$, $\sim 8.6 \%$ (mean: $5.8-30.8 \%$ ) for levels at $7-10 \%$, while it is highest (16.1-100\%; mean: 26.6\%) for levels >10\% [Kitzmiller et al., 1996]. Accordingly, in 2008, the UK Nation- 
Table 4. Proposed checklist for managing the reproductive risk of maternal diabetes

Preconception

- Reducing blood glucose and glycohemoglobin $(<6.1 \%)$ levels

- Controlling body weight $(\mathrm{BMI}<25)$

- Assuming high-dosage folic acid (5 mg/day)

- Limiting/avoiding the use of (potentially) teratogenic drugs related to common comorbitities (e.g. statins, ACE inhibitors)

1st trimester

- Ultrasound scan dating of pregnancy (starting at 8th week)

- Nuchal translucency and early gross malformations scan (starting at 10th week +6 days)

- Considering late 1st-trimester placental Doppler ultrasound

2nd trimester

- Maternal serum dosage of alpha-fetoprotein (16-18th week)

- Amniotic fluid dosage of alpha-fetoprotein in case of further indications for invasive techniques (e.g. advanced maternal age) (16-18th week)

- Ultrasound scanning for malformation in a specialized center (19-21th week)

- Fetal echocardiography

- Considering fetal MRI in case of ultrasound suspect and/or technical limitations to standard ultrasound scan (e.g. obesity, reduced amniotic fluid)

- Considering 2nd-trimester placental Doppler ultrasound

al Institute of Health and Clinical Excellence stated that glycohemoglobin levels below $6.1 \%$ associate with the lowest reproductive risk, while suggested to contraindicate pregnancy in women with glycohemoglobin levels above $10.0 \%$ [Kitzmiller et al., 2010]. All efforts should be applied to counsel women to start pregnancy with an optimal glycohemoglobin serum levels $<7 \%$ (optimal $<6.1 \%)$. The risk of major malformations is significantly higher in a population with glycohemoglobin levels above this cut-off and becomes prohibitive for levels $>10 \%$.

The contribution to adverse embryonic development of maternal prepregnancy obesity is difficult to dissect. Actually, the processes by which maternal overweight status may affect embryogenesis are still largely obscure. The most plausible mechanisms are those related to nutrition (i.e. teratogenic effect of deficiency and/or excess of specific nutrients) and to glycemic-control [Carmichael et al., 2010]. In both, obesity is only indirectly related to embryotoxicity, as it appears to be a maternal phenotypic feature coming together with the underlying teratogenic disorder. In addition, maternal obesity may interfere with the accuracy of prenatal ultrasound scan and, therefore, can reduce the chance of early detection of fetal malformations. In line with this, it should be recommended to start pregnancy with a body mass index $<25$.

It has been repeatedly demonstrated that maternal assumption of folic acid and antioxidant substances starting before fertilization reduces the rate of malformations in the offspring of diabetic mothers [Kitzmiller et al., 2010]. There is no international consensus on the most adequate daily intake of folic acid for prevention in diabetic mothers. In fact, while on one side the higher the quantity, the lower is the risk of malformations at birth, on the other, a daily assumption of $>1.000 \mu \mathrm{g}$ exposes women to the risk of vitamin $B_{12}$ deficiency, a not uncommon complication in maternal DM1 [Kitzmiller et al., 2010]. However, the prevalent recommendation consists of $5 \mathrm{mg} /$ day intake to be started at least 1 month before fertilization. As oxidative stress is a likely teratogenic mediator of maternal hyperglycemia in DM (see 'Mechanisms of Teratogenesis in Maternal Diabetes'), it is expected that preconception assumption of various antioxidant micronutrients can reduce the risk of malformations in diabetic pregnancies. Various studies confirm that the assumption of specific antioxidant substances, such as $\alpha$ lipoic acid [Sugimura et al., 2009], vitamin E [Cederberg et al., 2001; Gäreskog et al., 2006] and vitamin C [Cederberg et al., 2001], increases the chance of good prenatal outcome in the offspring of diabetic mothers. Therefore, in the near future, supplementation with multivitamins and fatty acids may become a further widely accepted resource for diabetic mothers [Reece, 2012].

With the increasing age at conception of prospective mothers and the wide repertoire of drugs used to treat common comorbidities at the time of fertilization, special care should be put on potential co-teratogenicity. In the last decades, the number of pregnant women with pre- 
existing DM2 is rising. In these patients the pros and cons of periconceptional use of statins should be carefully considered, even if a recent meta-analysis raised some concerns about the teratogenicity of early assumption of these drugs [Kusters et al., 2012]. Angiotensin-converting enzyme inhibitors and angiotensin receptor blockers, commonly used for treating systemic hypertension, are additional medications whose use should be avoided in pregnancy due to a strong teratogenic potential, which seems highest during the second and third trimesters [Polifka, 2012].

After fertilization, the effects of optimal glycemic and weight control have multifold beneficial effects on the pregnancy, as the potential effect of DM on the maternofetal unit is active during the entire pregnancy, although each complication shows different thresholds in relation to the developmental stage. During pregnancy, in addition to the medical follow-up, every pregnant woman with DM should undergo accurate monitoring of the in- tegrity of the embryofetal development and placental function by following a standardized schedule (table 4). In this scenario, the role of the clinical geneticist is expected to grow as the unique member of the prenatal medicine staff who has the prerequisites for coalescing specific competences in the fields of clinical embryology, communication and preventive medicine.

\section{Acknowledgements}

I wish to thank Prof. Mario Roggini (radiologist) and Dr. Evelina Silvestri (fetal-neonatal pathologist) for having so generously contributed to my cultural growth on embryopathology. With their efforts and enthusiasm, both handed down to me a sense of the essentialness of knowing both normal and abnormal intrauterine development in my job activity. The warmest thanks are for Prof. John M. Opitz, who - during the 14 days that I spent at the University of Utah (USA) and the many months fully dedicated to reading (and rereading) his monumental work - added an inestimable sprinkle of passion and mystery to this mixture.

\section{References}

Auerbach R: Analysis of the developmental effects of a lethal mutation in the house mouse. J Exp Zool 127:305-329 (1954).

Balsells M, García-Patterson A, Gich I, Corcoy R: Maternal and fetal outcome in women with type 2 versus type 1 diabetes mellitus: a systematic review and metaanalysis. J Clin Endocrinol Metab 94:4284-4291 (2009).

-Balsells M, García-Patterson A, Gich I, Corcoy R: Major congenital malformations in women with gestational diabetes mellitus: a systematic review and meta-analysis. Diabetes Metab Res Rev 28:252-257 (2012).

-Bánhidy F, Acs N, Puhó EH, Czeizel AE: Congenital abnormalities in the offspring of pregnant women with type 1 , type 2 and gestational diabetes mellitus: a populationbased case-control study. Congenit Anom (Kyoto) 50:115-121 (2010).

Barker DJ: The developmental origins of adult disease. J Am Coll Nutr 23 Suppl 6:588S595S (2004).

- Barr M Jr, Hanson JW, Currey K, Sharp S, Toriello $\mathrm{H}$, et al: Holoprosencephaly in infants of diabetic mothers. J Pediatr 102:565-568 (1983).

-Becerra JE, Khoury MJ, Cordero JF, Erickson JD: Diabetes mellitus during pregnancy and the risks for specific birth defects: a populationbased case-control study. Pediatrics 85:1-9 (1990).

Boulot P, Chabbert-Buffet N, d'Ercole C, Floriot $\mathrm{M}$, Fontaine $\mathrm{P}$, et al: French multicentric survey of outcome of pregnancy in women with pregestational diabetes. Diabetes Care 26: 2990-2993 (2003).
Boutte P, Valla JS, Lambert JC, Tordjman C, Berard E, Mariani R: The Vater association in a newborn infant of a diabetic mother [in French]. Pediatrie 40:219-222 (1985).

Brownlee M: Biochemistry and molecular cell biology of diabetic complications. Nature 414:813-820 (2001).

Carmichael SL, Rasmussen SA, Shaw GM: Prepregnancy obesity: a complex risk factor for selected birth defects. Birth Defects Res A Clin Mol Teratol 88:804-810 (2010).

Castori M, Rinaldi R, Capocaccia P, Roggini M, Grammatico P: VACTERL association and maternal diabetes: a possible causal relationship? Birth Defects Res A Clin Mol Teratol 82:169-172 (2008).

Castori M, Silvestri E, Cappellacci S, Binni F, Sforzolini GS, Grammatico P: Sirenomelia and VACTERL association in the offspring of a woman with diabetes. Am J Med Genet A 152A:1803-1807 (2010).

Cederberg J, Simán CM, Eriksson UJ: Combined treatment with vitamin $\mathrm{E}$ and vitamin $\mathrm{C}$ decreases oxidative stress and improves fetal outcome in experimental diabetic pregnancy. Pediatr Res 49:755-762 (2001).

Chen CP, Chen CY, Lin CY, Shaw SW, Wang W, Tzen CY: Prenatal diagnosis of concomitant alobar holoprosencephaly and caudal regression syndrome associated with maternal diabetes. Prenat Diagn 25:264-266 (2005).

Clausen TD, Mathiesen E, Ekbom P, Hellmuth E, Mandrup-Poulsen T, Damm P: Poor pregnancy outcome in women with type 2 diabetes. Diabetes Care 28:323-328 (2005)
Clausen TD, Mathiesen ER, Hansen T, Pedersen O, Jensen DM, et al: High prevalence of type 2 diabetes and pre-diabetes in adult offspring of women with gestational diabetes mellitus or type 1 diabetes: the role of intrauterine hyperglycemia. Diabetes Care 31: 340-346 (2008).

Comess LJ, Bennett PH, Burch TA, Miller M: Congenital anomalies and diabetes in the Prima Indians of Arizona. Diabetes 18:471477 (1969).

Correa A, Gilboa SM, Besser LM, Botto LD, Moore CA, et al: Diabetes mellitus and birth defects. Am J Obstet Gynecol 199:237.e1-9 (2008).

Crespi BJ: The emergence of human-evolutionary medical genomics. Evol Appl 4:292-314 (2011).

Croen LA, Shaw GM, Lammer EJ: Risk factors for cytogenetically normal holoprosencephaly in California: a population-based casecontrol study. Am J Med Genet 90:320-325 (2000)

Damasceno DC, Volpato GT, de Mattos Paranhos Calderon I, Cunha Rudge MV: Oxidative stress and diabetes in pregnant rats. Anim Reprod Sci 72:235-244 (2002).

Davis EM, Peck JD, Thompson D, Wild RA, Langlois P: Maternal diabetes and renal agenesis/dysgenesis. Birth Defects Res A Clin Mol Teratol 88:722-727 (2010).

De Hertogh R, Vanderheyden I, Pampfer S, Robin D, Dufrasne E, Delcourt J: Stimulatory and inhibitory effects of glucose and insulin on rat blastocyst development in vitro. Diabetes 40:641-647 (1991) 
Dentici ML, Placidi S, Francalanci P, Capolino R, Rinelli G, et al: Association of DiGeorge anomaly and caudal dysplasia sequence in a neonate born to a diabetic mother. Cardiol Young 6:1-4 (2012).

Deuchar EM: Embryonic malformations in rats, resulting from maternal diabetes: preliminary observations. J Embryol Exp Morphol 41:93-99 (1977).

-de Valk HW, van Nieuwaal NH, Visser GH: Pregnancy outcome in type 2 diabetes mellitus: a retrospective analysis from the Netherlands. Rev Diabet Stud 3:134-142 (2006).

-Digilio MC, Marino B, Formigari R, Giannotti A: Maternal diabetes causing DiGeorge anomaly and renal agenesis. Am J Med Genet 55:513-514 (1995).

Donat JF: Septo-optic dysplasia in an infant of a diabetic mother. Arch Neurol 38:590-591 (1981).

Driscoll SG: The pathology of pregnancy complicated by diabetes mellitus. Med Clin North Am 49:1053-1067 (1965).

D Dudley DJ: Diabetic-associated stillbirth: incidence, pathophysiology, and prevention. Obstet Gynecol Clin North Am 34:293-307 (2007).

Duncan PA, Shapiro LR, Klein RM: Sacrococcygeal dysgenesis association. Am J Med Genet 41:153-161 (1991).

-el-Hage S, Singh SM: Temporal expression of genes encoding free radical-metabolizing enzymes is associated with higher mRNA levels during in utero development in mice. Dev Genet 11:149-159 (1990).

Eriksson UJ: Diabetes in pregnancy: effects on post-implantation embryos. Isr J Med Sci 27: 478-486 (1991).

- Eriksson UJ, Bone AJ, Turnbull DM, Baird JD: Timed interruption of insulin therapy in diabetic $\mathrm{BB} / \mathrm{E}$ rat pregnancy: effect on maternal metabolism and fetal outcome. Acta Endocrinol (Copenh) 120:800-810 (1989).

Evers IM, de Valk HW, Visser GH: Risk of complications of pregnancy in women with type 1 diabetes: nationwide prospective study in the Netherlands. BMJ 328:915 (2004).

Ewart-Toland A, Yankowitz J, Winder A, Imagire R, Cox VA, et al: Oculoauriculovertebral abnormalities in children of diabetic mothers. Am J Med Genet 90:303-309 (2000).

-Fernández-Morera JL, Rodríguez-Rodero S, Menéndez-Torre E, Fraga MF: The possible role of epigenetics in gestational diabetes: cause, consequence, or both. Obstet Gynecol Int 2010:605163 (2010).

- Fine EL, Horal M, Chang TI, Fortin G, Loeken MR: Evidence that elevated glucose causes altered gene expression, apoptosis, and neural tube defects in a mouse model of diabetic pregnancy. Diabetes 48:2454-2462 (1999).

- Frías JL, Frías JP, Frías PA, Martínez-Frías ML: Infrequently studied congenital anomalies as clues to the diagnosis of maternal diabetes mellitus. Am J Med Genet A 143A:29042909 (2007).

Diabetic Embryopathy: A Developmental Perspective

\section{Perspective}

Gäreskog M, Eriksson UJ, Wentzel P: Combined supplementation of folic acid and vitamin $\mathrm{E}$ diminishes diabetes-induced embryotoxicity in rats. Birth Defects Res A Clin Mol Teratol 76:483-490 (2006).

- Gosseye S, Golaire MC, Verellen G, Van Lierde $\mathrm{M}$, Claus D: Association of bilateral renal agenesis and Di George syndrome in an infant of a diabetic mother. Helv Paediatr Acta 37:471-474 (1982)

Greene MF, Hare JW, Cloherty JP, Benacerraf BR, Soeldner JS: First-trimester hemoglobin $\mathrm{A} 1$ and risk for major malformation and spontaneous abortion in diabetic pregnancy. Teratology 39:225-231 (1989).

- Gripp KW, Barr M Jr, Anadiotis G, McDonaldMcGinn DM, Zderic SA, Zackai EH: Aphallia as part of urorectal septum malformation sequence in an infant of a diabetic mother. Am J Med Genet 82:363-367 (1999).

Hall BD: VATER/VACTERL association, in Cassidy SB, Allanson JE (eds): Management of Genetic Syndromes, ed 3, pp 871-880 (Wiley-Bleckwell, Hoboken 2010).

-Hanson U, Persson B: Outcome of pregnancies complicated by type 1 insulin-dependent diabetes in Sweden: acute pregnancy complications, neonatal mortality and morbidity. Am J Perinatol 10:330-333 (1993).

-Hod M, Star S, Passonneau JV, Unterman TG, Freinkel N: Effect of hyperglycemia on sorbitol and myo-inositol content of cultured rat conceptus: failure of aldose reductase inhibitors to modify myo-inositol depletion and dysmorphogenesis. Biochem Biophys Res Commun 140:974-980 (1986)

Horii KI, Watanabe GI, Ingalls TH: Experimental diabetes in pregnant mice. Prevention of congenital malformations in offspring by insulin. Diabetes 15:194-204 (1966).

Hunter ES 3rd, Sadler TW: Fuel-mediated teratogenesis: biochemical effects of hypoglycemia during neurulation in mouse embryos in vitro. Am J Physiol 257:E269-276 (1989).

Ishibashi M, Akazawa S, Sakamaki H, Matsumoto K, Yamasaki H, et al: Oxygen-induced embryopathy and the significance of glutathione-dependent antioxidant system in the rat embryo during early organogenesis. Free Radic Biol Med 22:447-454 (1997).

- Jain SK, McVie R, Duett J, Herbst JJ: Erythrocyte membrane lipid peroxidation and glycosylated hemoglobin in diabetes. Diabetes 38: 1539-1543 (1989).

James AW, Culver K, Hall B, Golabi M: Bifid tongue: a rare feature associated with infants of diabetic mother syndrome. Am J Med Genet A 143A:2035-2039 (2007).

Jensen DM, Damm P, Moelsted-Pedersen L, Ovesen P, Westergaard JG, et al: Outcomes in type 1 diabetic pregnancies: a nationwide, population-based study. Diabetes Care 27: 2819-2823 (2004)

Johnson JP, Carey JC, Gooch WM 3rd, Petersen J, Beattie JF: Femoral hypoplasia-unusual facies syndrome in infants of diabetic mothers. J Pediatr 102:866-872 (1983).
Joss-Moore LA, Lane RH: The developmental origins of adult disease. Curr Opin Pediatr 21:230-234 (2009).

-Kalter H: Diabetes and spontaneous abortion: a historical review. Am J Obstet Gynecol 156: 1243-1253 (1987).

Kalter H: Case reports of malformations associated with maternal diabetes: history and critique. Clin Genet 43:174-179 (1993).

-Kitzmiller JL, Buchanan TA, Kjos S, Combs CA, Ratner RE: Pre-conception care of diabetes, congenital malformations, and spontaneous abortions. Diabetes Care 19:514-541 (1996).

- Kitzmiller JL, Wallerstein R, Correa A, Kwan S Preconception care for women with diabetes and prevention of major congenital malformations. Birth Defects Res A Clin Mol Teratol 88:791-803 (2010).

Kucera J: Rate and type of congenital anomalies among offspring of diabetic women. J Reprod Med 7:73-82 (1971)

Kusters DM, Lahsinoui HH, van de Post JA, Wiegman A, Wijburg FA, et al: Statin use during pregnancy: a systematic review and meta-analysis. Expert Rev Cardiovasc Ther 10:363-378 (2012).

- Lauenborg J, Mathiesen E, Ovesen P, Westergaard JG, Ekbom P, et al: Audit on stillbirths in women with pregestational type 1 diabetes. Diabetes Care 26:1385-1389 (2003).

Li R, Chase M, Jung SK, Smith PJ, Loeken MR: Hypoxic stress in diabetic pregnancy contributes to impaired embryo gene expression and defective development by inducing oxidative stress. Am J Physiol Endocrinol Metab 289:E591-E599 (2005).

Lisowski LA, Verheijen PM, Copel JA, Kleinman CS, Wassink S, et al: Congenital heart disease in pregnancies complicated by maternal diabetes mellitus. An international clinical collaboration, literature review, and metaanalysis. Herz 35:19-26 (2010).

Loffredo CA, Wilson PD, Ferencz C: Maternal diabetes: an independent risk factor for major cardiovascular malformations with increased mortality of affected infants. Teratology 64:98-106 (2001)

Macintosh MC, Fleming KM, Bailey JA, Doyle P, Modder J, et al: Perinatal mortality and congenital anomalies in babies of women with type 1 or type 2 diabetes in England, Wales, and Northern Ireland: population based study. BMJ 333:177 (2006).

Martínez-Frías ML: Epidemiological analysis of outcomes of pregnancy in diabetic mothers: identification of the most characteristic and most frequent congenital anomalies. Am J Med Genet 51:108-113 (1994)

Melamed N, Hod M: Perinatal mortality in pregestational diabetes. Int J Gynaecol Obstet 104 Suppl 1:S20-S24 (2009)

-Miller EA, Rasmussen SA, Siega-Riz AM, Frías JL, Honein MA; National Birth Defects Prevention Study: Risk factors for non-syndromic holoprosencephaly in the National Birth Defects Prevention Study. Am J Med Genet C Semin Med Genet 154C:62-72 (2010). 
-Miller JF, Williamson E, Glue J, Gordon YB, Grudzinskas JG, Sykes A: Fetal loss after implantation. A prospective study. Lancet 2: 554-556 (1980).

Mills JL, Simpson JL, Driscoll SG, Jovanovic-Peterson L, Van Allen M, et al: Incidence of spontaneous abortion among normal women and insulin-dependent diabetic women whose pregnancies were identified within 21 days of conception. N Engl J Med 319:16171623 (1988).

-Mimouni F, Miodovnik M, Siddiqi TA, Khoury J, Tsang RC: Perinatal asphyxia in infants of insulin-dependent diabetic mothers. J Pediatr 113:345-353 (1988).

- Novak RW, Robinson HB: Coincident DiGeorge anomaly and renal agenesis and its relation to maternal diabetes. Am J Med Genet 50: 311-312 (1994).

-Opitz JM: Blastogenesis and the 'primary field' in human development. Birth Defects Orig Artic Ser 29:3-37 (1993).

-Opitz JM, Zanni G, Reynolds JF Jr, Gilbert-Barness E: Defects of blastogenesis. Am J Med Genet 115:269-286 (2002).

Ornoy A, Zusman I: Embryotoxic effects of diabetes on pre-implantation embryos. Isr J Med Sci 27:487-492 (1991).

- Pampfer S, de Hertogh R, Vanderheyden I, Michiels B, Vercheval M: Decreased inner cell mass proportion in blastocysts from diabetic rats. Diabetes 39:471-476 (1990).

- Pani L, Horal M, Loeken MR: Polymorphic susceptibility to the molecular causes of neural tube defects during diabetic embryopathy. Diabetes 51:2871-2874 (2002a).

- Pani L, Horal M, Loeken MR: Rescue of neural tube defects in Pax-3-deficient embryos by p53 loss of function: implications for Pax-3dependent development and tumorigenesis. Genes Dev 16:676-680 (2002b).

- Pedersen J, Mølsted-Pedersen LM: Congenital malformations: the possible role of diabetes care outside pregnancy. Ciba Found Symp 63:265-271 (1978).
Phelan SA, Ito M, Loeken MR: Neural tube defects in embryos of diabetic mice: role of the Pax-3 gene and apoptosis. Diabetes 46:11891197 (1997).

Platt MJ, Stanisstreet M, Casson IF, Howard CV, Walkinshaw S, et al: St Vincent's Declaration 10 years on: outcomes of diabetic pregnancies. Diabet Med 19:216-220 (2002).

Polifka JE: Is there an embryopathy associated with first-trimester exposure to angiotensin-converting enzyme inhibitors and angiotensin receptor antagonists? A critical review of the evidence. Birth Defects Res A Clin Mol Teratol 94:576-598 (2012).

Reece EA: Diabetes-induced birth defects: what do we know? What can we do? Curr Diab Rep 12:24-32 (2012).

Reece EA, Homko CJ, Wu YK: Multifactorial basis of the syndrome of diabetic embryopathy. Teratology 54:171-182 (1996).

-Salbaum JM, Kappen C: Neural tube defect genes and maternal diabetes during pregnancy. Birth Defects Res A Clin Mol Teratol 88:601611 (2010).

Salbaum JM, Kappen C: Diabetic embryopathy: a role for the epigenome? Birth Defects Res A Clin Mol Teratol 91:770-780 (2011).

-Salbaum JM, Kappen C: Responses of the embryonic epigenome to maternal diabetes. Birth Defects Res A Clin Mol Teratol 94:770-781 (2012).

-Smoak IW, Sadler TW: Embryopathic effects of short-term exposure to hypoglycemia in mouse embryos in vitro. Am J Obstet Gynecol 163:619-624 (1990).

-Solomons NW: Developmental origins of health and disease: concepts, caveats, and consequences for public health nutrition. Nutr Rev 67 Suppl 1:S12-S16 (2009).

-Sugimura Y, Murase T, Kobayashi K, Oyama K, Hayasaka S, et al: Alpha-lipoic acid reduces congenital malformations in the offspring of diabetic mice. Diabetes Metab Res Rev 25: 287-294 (2009).

-Sussman I, Matschinsky FM: Diabetes affects sorbitol and myo-inositol levels of neuroectodermal tissue during embryogenesis in rat. Diabetes 37:974-981 (1988).
Uriu-Hare JY, Stern JS, Keen CL: Influence of maternal dietary $\mathrm{Zn}$ intake on expression of diabetes-induced teratogenicity in rats. Diabetes 38:1282-1290 (1989).

Valenzano M, Paoletti R, Rossi A, Farinini D, Garlaschi G, Fulcheri E: Sirenomelia. Pathological features, antenatal ultrasonographic clues, and a review of current embryogenic theories. Hum Reprod Update 5:82-86 (1999).

-Vaz SS, Chodirker B, Prasad C, Seabrook JA, Chudley AE, Prasad AN: Risk factors for nonsyndromic holoprosencephaly: a Manitoba case-control study. Am J Med Genet A 158A:751-758 (2012).

-Wang R, Martínez-Frías ML, Graham JM Jr: Infants of diabetic mothers are at increased risk for the oculo-auriculo-vertebral sequence: a case-based and case-control approach. J Pediatr 141:611-617 (2002).

-Watanabe G, Ingalls TH: Congenital malformations in the offspring of alloxan-diabetic mice. Diabetes 12:66-72 (1963).

White P: Pregnancy complicating diabetes, in Joslin EP, Root HF, White P, Marble A (eds): The Treatment of Diabetes Mellitus, pp 633638 (Lea \& Febiger, Philadelphia 1937).

-Wilson TA, Blethen SL, Vallone A, Alenick DS, Nolan P, et al: DiGeorge anomaly with renal agenesis in infants of mothers with diabetes. Am J Med Genet 47:1078-1082 (1993).

Yajnik CS: Nutrient-mediated teratogenesis and fuel-mediated teratogenesis: two pathways of intrauterine programming of diabetes. Int J Gynaecol Obstet 104 Suppl 1:S27-S31 (2009).

Yogev Y, Chen R, Ben-Haroush A, Hod M, Bar J: Maternal overweight and pregnancy outcome in women with Type-1 diabetes mellitus and different degrees of nephropathy. J Matern Fetal Neonatal Med 23:999-1003 (2010).

Zabihi S, Loeken MR: Understanding diabetic teratogenesis: where are we now and where are we going? Birth Defects Res A Clin Mol Teratol 88:779-790 (2010). 